tortoiseshell males. When further results become available it is hoped to deal with these and other matters in detail.

3 Hill View Road, Mapperley, Notts.

A. C. JUdE

Department of Zoology,

University of Malaya,

Singapore 10.

1 Little, C. C., Science, N.S., 35, 784 (1912). Doncaster, L., ibid., 86, 144 (1912).

'Bamber, R., Bib. Genetica, 3, 1 (1927). Komal, T., Proc. Japan Acad., 28, 150 (1952).

3 Doncaster, L., J. Genet., 3, 11 (1913).

4 Komal, T., $J$. Hered. (in the press) and personal communication,

- Jude, A. C., Nature, 172, 81 (1953). Searle, A. G., and Jude, A. C., J. Genet., 54, 506 (1956).

\section{Shape Discrimination in the Octopus and the Rat}

EXPERIMENTS on shape discrimination in rats, using the Lashley two-card jumping stand, have shown that, when rats are pre-trained to discriminate horizontal from vertical striations (horizontal positive), they tend to choose other shapes with a horizontal base-line, such as a square, triangle or pentagon, in preference to a second shape which lacks the horizontal base, such as a circle or inverted triangle. This suggests that a horizontal line in one shape of a pair which have to be discriminated from one another is isolated by the animal as a basis for discrimination; this has been verified for a number of 'transfer' shapes, and in each case the horizontal base was the effective element in discrimination. These experiments, reported in detail elsewhere ${ }^{1}$, were designed to overcome objections to earlier work in this field, for example, the classic studies of Lashley ${ }^{2}$.

N. S. Sutherland ${ }^{3}$ has shown that horizontal and vertical components of shapes are also important in shape recognition in the octopus. He suggests a theory of shape recognition to account for the importance of these components in discriminations by visually primitive organisms. I have put forward a similar theory which also accounts for this phenomenon; both theories would appear prima facie to account equally well for both sets of data. On closer inspection, however, Sutherland's theory shows a considerable weakness, at least in the form so far reported. In his theory, discrimination is based on a comparison between the separate outputs from the rows and columns of an array of cells (cf. Sutherland's diagrams), a comparison which involves both the number of cells active, and the intensity of their firing in the row output, with the same characteristics (of number and intensity) in the column output.

It seems plausible to suppose that an 'analysing mechanism' could be devised to compare these quantities when their difference is relatively large (in Sutherland's Fig. 1, one fibre firing at intensity five, compared with five fibres at intensity one). As soon as the outputs become more complex, for example, the third pair of figures in Sutherland's Fig. 2, the theory becomes more difficult to accept. In this instance the analysing mechanism has to detect a spatial relationship among the fibres which are active, irrespective of the position which the set of active fibres occupies in the cable. This difficulty is further enhanced for the shapes shown in his Fig. 3. Here, in three cases, the output is identical in all respects for rows and columns, and hence a comparison of row and column outputs for a single shape no longer provides a distinctive characteristic for that shape; discrimination must depend on comparison of the output for one shape with the output for another. Hence the theory can only function if an analysing mechanism is suggested which can perform such comparisons.

Unfortunately, Sutherland does not say what characteristics this analyser has which enables it to detect differences in the row and column outputs for different shapes, although it is clear that these characteristics are of fundamental importance for the theory. If Sutherland has devised an analysing mechanism with the requisite properties, he has made a contribution to the theory of shape discrimination, since the classifying of a spatial pattern irrespective of its location has always been a stumbling block for neural theories of shape perception. With such an analysing mechanism, however, the reason for postulating the array of cells becomes obscure, because the property by virtue of which the analyser must (apparently) work in his theory should enable it to deal directly with information from the retina (in terms of spatial patterns of excitation) without the intermediating array.

My own theory ${ }^{1}$ overcomes this difficulty of recognizing spatial patterns of excitation by. introducing a transformation into a temporal pattern, which is transmitted in the output cables for rows and columns of an array of cells similar to that postulated by Sutherland.

Department of Psychology,

Birkbeck College,

Malet Street,

London, W.C.1.

1 Dodwell, P. C., Brit. J. Psychol. (in the press).

2 Lashley, K. S., J. Gen. Psychol., 18, 123 (1938).

${ }^{3}$ Sutherland, N. S., Nature, 179, 11 (1957).

\section{Proposed Nomenclature in the Field of Neuraminic and Sialic Acids}

IT would appear that the chemical relationship of the substances designated by various names such as neuraminic, sialic, lactiminic and gynaminic acid has been established. In order to avoid further confusion we propose to call the basic, unsubstituted compound neuraminic acid. Sialic acid is suggested as group name for the acylated neuraminic acids (for example, N-acetylneuraminic acid, N-glycolylneuraminic acid, diacetylneuraminic acids). For the enzyme which splits the glycosidic linkage joining the terminal sialic acid to the residual oligo- or polysaccharide the names neuraminidase and sialidase may be used synonymously. We have agreed to use this nomenclature in future.

Department of Medical Chemistry,

$$
\text { F. G. Burx }
$$

University of Upsala.

\section{A. Gotrschalk}

Walter and Eliza Hall

Institute of Medical Research, Melbourne.

\section{E. KLENK}

Department of Physiological Chemistry,

University of Cologne. April 24. 